\title{
Pedestrian Evacuation Model considering Dynamic Emotional Update in Direction Perception Domain
}

\author{
Qian Xiao (iD) ${ }^{1}$ and Jiayang $\mathrm{Li}$ (iD) ${ }^{1,2}$ \\ ${ }^{1}$ School of Information Engineering, Shenyang University, Shenyang, Liaoning 110044, China \\ ${ }^{2}$ School of Business Administration, Northeastern University, Shenyang, Liaoning 110000, China \\ Correspondence should be addressed to Qian Xiao; xiaoqian_neu@163.com
}

Received 16 February 2021; Revised 29 May 2021; Accepted 25 August 2021; Published 1 September 2021

Academic Editor: Atila Bueno

Copyright (c) 2021 Qian Xiao and Jiayang Li. This is an open access article distributed under the Creative Commons Attribution License, which permits unrestricted use, distribution, and reproduction in any medium, provided the original work is properly cited.

\begin{abstract}
Traditional dynamic models cannot fully describe the microdetails of the impact of emotional contagion on individual state and behavior when pedestrian evacuation is simulated. This paper addresses the problem by constructing a dynamic evolution mechanism among emotion, state, and behavior. First, the direction perception domain of pedestrians during evacuation is defined. Then, the dynamic emotional perception of pedestrians during an evacuation is studied, considering the emotional increment caused by personal walking speed and others in the direction perception domain. Next, emotional contagion is introduced into the improved cellular automata (CA) simulation model in the floor field (FF), entitled the "CECA model." The transfer probability of pedestrians in different states is proposed by defining "susceptible emotional state" and "infectious emotional state." Finally, the simulation results are compared with known models. The results demonstrate that the improved model can improve the evacuation efficiency of the system significantly. Simultaneously, the effects of emotional threshold, infection coefficient, calm coefficient, and perception radius of the evacuation system on the pedestrian evacuation process are simulated and analyzed, providing a basis for evacuation managers to formulate evacuation strategies.
\end{abstract}

\section{Introduction}

The dynamics of pedestrian emergency evacuation in public places has become a popular topic. How to model pedestrian evacuation and optimize pedestrian evacuation strategy scientifically is particularly important. At present, there are many achievements in the simulation of the pedestrian evacuation model. The commonly used evaluation models include macro- and microevaluation models. The macromodel predominantly studies the characteristics of pedestrian movement, bottleneck problem, and cluster phenomenon. In contrast, the micromodel considers more details on pedestrian movement or human evacuation behavior in different environments. In recent years, it has become the focus of pedestrian evacuation research to study the microbehavior characteristics of pedestrians by simulating real scenarios with a simulation model. Standard simulation models include the cellular automata (CA) model, social force model, multigrid model, lattice gas model, and visual obstacle model [1-5].

In 1997, Blue et al. [6] proposed the CA model and applied it to pedestrian traffic research. The advantage of the CA model is that it has a strong ability to simulate the spatiotemporal dynamic evolution of a spatial complex system and requires lower computation. Burstedde et al. [7] and Schadschneider [8] introduced the concept of static and dynamic "floor field" (FF) in the CA model and constructed a field CA model to study the evacuation characteristics of pedestrian flow. Fang et al. [9] discussed various possibilities for pedestrians to move next to the moving neighborhood, which further enriched the CA model. Yang et al. [10] used CA to simulate the pedestrian countercurrent phenomenon in the passage by introducing the right preference of pedestrian walking. Yue et al. [11] simulated the dynamic characteristics of pedestrian evacuation by building a dynamic parameter model. Li et al. [1] proposed a CA model to 
study the potential visibility of pedestrians in the dark. Ji et al. [12] proposed a new triangular mesh CA model to solve the problem of high-density crowd evacuation. Zheng et al. [13] proposed an extended FF model to study the effect of three-dimensional smoke diffusion on pedestrian evacuation. Shi et al. [14] proposed a new impatience judgment model to qualitatively and quantitatively describe the impatience degree during the evacuation and applied it to the CA model to verify the results. Geng et al. [15] proposed an extended CA model to simulate pedestrian evacuation under adverse line-of-sight conditions by considering the influence of uncertain information.

During the emergency, tension will affect people's judgment and behavior decisions [16]. Mastering the emotions of pedestrians and guiding them is an effective way to avoid injury. Many researchers have conducted in-depth research on the emotion and behavior of pedestrian evacuation. The ocean model proposed by Costa and McCrae [17] is the most widely used personality model. Helbing [18] simulated the dynamic behavior of pedestrians fleeing in an emergency, concluding that panic fleeing behavior is the behavioral representation of tension. David et al. [19] concluded through empirical research that pedestrians walking under a relevant emotional state could have their risk perception ability negatively affected, with the potential consequences of suffering road accidents. How to express the alternation and dynamic evolution of emotion and embed the dynamic state of emotion into the pedestrian movement model is a complex problem when constructing an evacuation simulation model. Fu et al. [20] used a multigrid model to understand pedestrian dynamics in counterflow, coupled with the effect of emotional propagation in a crowd stampede induced by panic. Chen et al. [21] proposed an extended FF CA model considering pedestrian panic to investigate pedestrian dynamics under the threat of artificial attack. Zheng et al. [22] proposed an improved CA model of FF to study pedestrian evacuation in an emergency by considering the diffusion of emergency and pedestrian tension. Li et al. [23] proposed an extended cost potential field CA to simulate pedestrian counterflow under an emergency by considering behavior variation in pedestrians induced by psychological tension.

In the process of pedestrian evacuation, tension not only affects individuals but also spreads through emotional infection among individuals. Cocking et al. [24] pointed out through empirical analysis that emotions can spread rapidly among people during an emergency evacuation, even among strangers. Fu et al. [25] suggested that there was contact infection in pedestrian emotion. Nilsson and Johansson [26] used empirical experiments to confirm that pedestrian emotion and evacuation behavior are more affected by close distance than by long distance. Mao et al. [27] demonstrated an emotional contagion model based on intimacy to simulate peer decision-making during the emergency evacuation.

This paper analyzes the impact of tension on pedestrian evacuation from the perspective of personality. The human study of group emotional contagion originated from infectious diseases. It is a scientific infectious disease model for exploring how diseases spread among groups. There are two typical models: SIR and SIS. The SIR model refers to three types of individual statuses: susceptible, infectious, and removal [28]. Fu et al. [25] proposed a new emotional contagion model (CA-SIRS) by combining the epidemiological SIR model with CA. However, the SIR model does not consider the infection of some diseases, so it is difficult for individuals to achieve complete immunity. Therefore, the SIS emotional infection model proposes that there are only susceptible and infectious people during infection [29]. Cao et al. [30] constructed an emotional contagion model based on the SIS and social force models to reflect the diversity of individuals in the group. However, the infectious disease model is macromathematical. Most of the research is still focused on the macrocharacteristics and qualitative analysis of emotion and emotional contagion while ignoring microdetails such as the impact of emotional contagion on the states of evacuation individual and the possible types of individual movement in different emotional states.

During pedestrian movement, heterogeneity of emotional contagion occurs due to walking direction, visual obstacles, and other factors. An improved micromodel of the dynamic emotion and infection mechanism based on the theory of emotional communication and infectious dynamics is constructed in this paper to examine the pedestrian evacuation problem considering emotional contagion thoroughly. First, the emotional perception domain is defined. Then, the dynamic emotional perception of pedestrians in evacuation is analyzed from two aspects: the change in individual speed and the different perceived distances in the direction perception domain. The remainder of this paper is structured as follows. In Section 2, the macro-SIS model is modified to the micro CECA model, and the emotional state of evacuation individuals is defined as two types: "susceptible emotional state" and "infectious emotional state." A dynamic pedestrian evacuation model combined with CA considering emotional contagion is constructed, and the transfer probability is improved. Then, in Section 3, the model's effectiveness is analyzed by simulating the evacuation process. The influence of the emotional threshold, infection coefficient, calm coefficient, and perception radius on evacuation efficiency and the change in crowd evacuation state under different emotions are discussed. Finally, the research is summarized in the Section 4 .

\section{Dynamic Emotional Perception considering Direction Perception Domain}

2.1. Definition of Direction Perception Domain. In the process of pedestrian evacuation, tension among many people can be contagious. Pedestrians will recognize, feel, and be affected by the emotions of others around them [18]. For studying the differences in pedestrian emotion affected by different locations around the pedestrian, this paper defines the direction perception domain.

This paper describes the pedestrian's position and surrounding neighborhood during an evacuation using the Moore neighborhood in the traditional CA model [9-11], which can describe the pedestrian's evacuation behavior 
effectively. Based on the Moore neighborhood, this paper defines evacuation simulation scenarios in independent space. The space is $L$ in length and $W$ in width. It is divided into a certain number of cell lattices of $0.4 \times 0.4$. Each cell is the floor area of a person standing, which is represented by a location coordinate cell $(x, y)$. The distance between adjacent cells is $c l=0.4 \mathrm{~m}$. The number of exits is $m$, and the width is $e l_{i}$, where $i \in[1,2, \ldots, m]$. When the initial state $t=0$, pedestrians are randomly distributed in the space, each occupying a cell. After the evacuation, the Moore neighborhood rule is adopted. Each time, the pedestrian determines the next move strategy according to the cell's transfer probability in each direction until the pedestrian reaches the exit and escape.

$\mathrm{Fu}$ et al. [25] considers that the emotional perception range of pedestrian after discretization is a square with $R$ as the radius and the pedestrian cell $j$ as the center. However, there are differences in a pedestrian's judgment of different directions due to the limitation of the pedestrian's line-ofsight. Therefore, the influence of information transmission in line-of-sight differs from that in nonline-of-sight. Accordingly, the perception domain is divided into visual and nonvisual, as depicted in Figure 1.

The visual perception domain $R V$ is defined as the discretized region with the cell coordinate cell $\left(x_{j}, y_{j}\right)$ of evacuation individual $j$ at time $t$ as the center, $\left[\theta_{j}(t)-\right.$ $\left.(\pi / 4), \theta_{j}(t)+(\pi / 4)\right]$ as the angle, and $R$ as the radius.

The nonvisual perception domain $R I$ is defined as the discretized region with the cell coordinate cell $\left(x_{j}, y_{j}\right)$ of evacuation individual $j$ at time $t$ as the center and $R$ as the radius, excluding the domain $R V$.

The evacuation individuals and direction perception domains in different forward directions at time $t$ are depicted in Figure 2.

Pedestrians are more sensitive to the emotional perception of nearby areas during an evacuation [25, 31]. Therefore, this paper also considers that the influence of others' emotions on individual $j$ is different in visual and nonvisual perception domains. Combined with the distance factor, this paper considers that the perceived degree of others' emotions at a close distance is greater than that at a long distance in the visual perception domain. Furthermore, the perceived degree of others' emotions in the visual perception domain is greater than that in the nonvisual perception domain.

2.2. Dynamic Emotional Perception Based on Direction Perception Domain. Cocking et al. [24] believe that the emotional value of evacuation individuals is related to their self emotions and the emotions of others around them. In this paper, the dynamic equation of the tension emotional intensity of pedestrian $j$ at time $t$ is defined by

$$
E_{j}(t)=E_{\text {self }}(t)+E_{\mathrm{DPD}}(t)+\tau,
$$

where $E_{\text {self }}(t)$ is the self emotional intensity of evacuation individual $j$ at time $t, E_{\mathrm{DPD}}(t)$ is the emotional intensity change of others perceived by evacuation individual $j$ at time

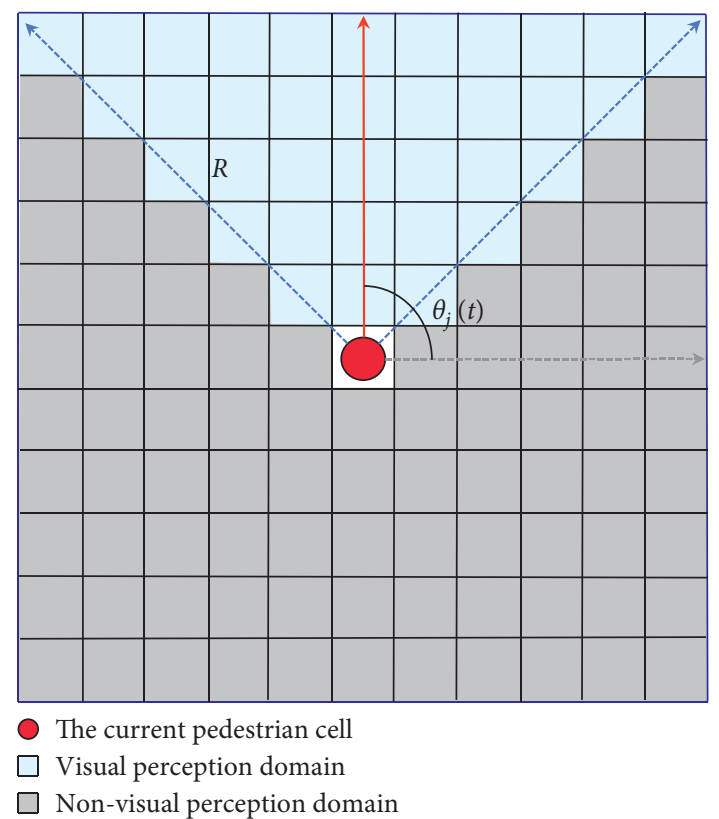

FIgURE 1: Visual and nonvisual perception domains with $R=2 \mathrm{~m}$.

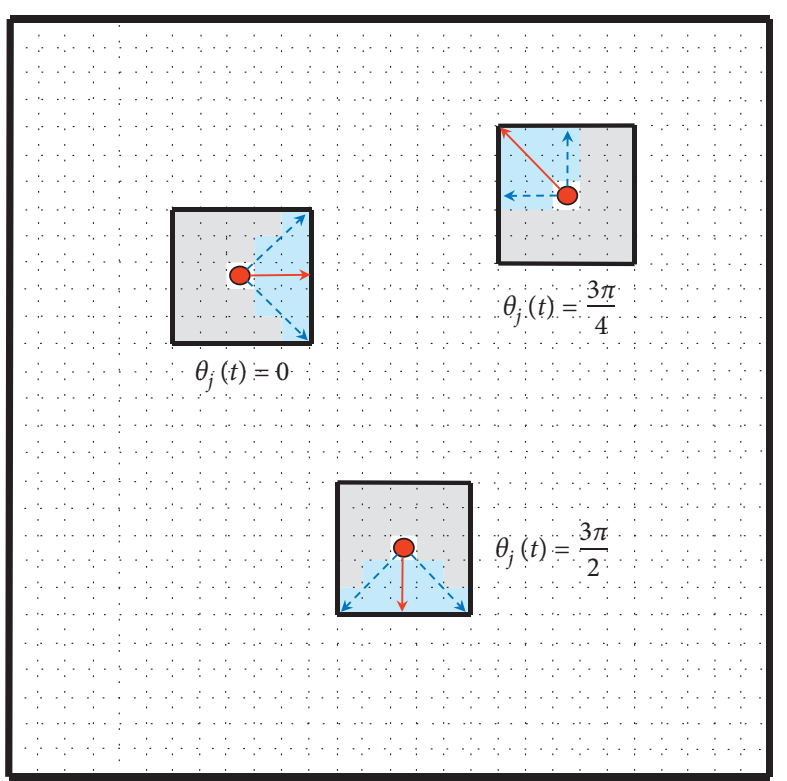

FIgUre 2: Evacuate individuals and direction perception domains at time $t$.

$t, \tau$ is the random disturbance, and the value range of $E_{j}(t)$ is $(0,1]$.

2.2.1. The Self Emotional Intensity $E_{\text {self }}(t)$ of Evacuation Individual $j$ at Time $t$. This paper considers that the self emotional intensity of evacuation individual $j$ at time $t$ is based on the emotional intensity at time $t-1$ and then superimposed with the emotional perception increment caused by speed. During an evacuation, if the individual's walking speed is lower than the maximum expected speed, his negative emotion increases rapidly. Moreover, if the 
individual's walking speed is close to the maximum expected speed, the growth of his negative emotion is relatively slow [11]. Thus, define the ratio of the speed at the previous time to the maximum expected speed, expressed as $v f=\left(v_{j}(t-1) / v_{j}^{\max }\right)$. Therefore, the self emotional intensity of evacuation individual $j$ at time $t$ is depicted in

$$
E_{\text {self }}(t)=E_{j}(t-1)+(1-v f) e^{-\alpha \cdot v f},
$$

where $E_{j}(t-1)$ is the self emotional intensity at time $t-1, \alpha$ is the speed-sensitive parameter of individual $j$ at $t$, and the value range is $[0,1]$.

Equation (3) calculates the fluctuation of pedestrian emotion caused by the change in speed precisely. If the actual speed is close to the maximum expected speed, that is, $v f$ is close to 1 , the tension emotion of evacuation individuals fluctuates less. If the actual speed is far less than the maximum expected speed, that is, $v f \ll 1$, the tension emotion of evacuation individuals fluctuates greatly.

\subsubsection{The Definition of Emotional Increment $E_{D P D}(t)$ Caused} by Others in Direction Perception Domain. In the direction perception domain, different distances have different influences on emotion. Zheng et al. [22] proposed that distance can influence weight in the emotional gain function. Suppose that cell $\left(x_{j}, y_{j}\right)$ is the location coordinate of pedestrian cell $j$ and cell $\left(x_{k}, y_{k}\right)$ is the location coordinate of target cell $k$. The distance between cells adopts Euclidean distance. Then, the distance between pedestrian cell $j$ and target cell $k$ is as shown in

$$
d_{j k}=d\left(x_{j}, y_{j}, x_{k}, y_{k}\right) \cdot c l=\sqrt{\left(x_{j}-x_{k}\right)^{2}+\left(y_{j}-y_{k}\right)^{2}} \cdot c l,
$$

where $c l$ is the distance between adjacent cells.

This paper reflects the difference in the perception degree in the perception domain by redefining the distance weight, as shown in

$$
D_{j k}=\left[1-\left(1+e^{-d_{j k}}\right)^{-1}\right] \cdot\left\{\sum\left[1-\left(1+e^{-d_{j k}}\right)^{-1}\right]\right\}^{-1} .
$$

When individual cells perceive the emotional difference with others at the previous moment, they will be affected and have emotional changes. The emotional difference is expressed as $\Delta E_{j k}(t-1)=E_{k}(t-1)-E_{j}(t-1)$. Therefore, based on the distance weight, the average change function of others' emotions perceived by individual cell $j$ at time $t$ is defined as follows:

$$
\begin{aligned}
E_{\mathrm{DPD}}(t)= & \left(n_{R V}\right)^{-1} \sum_{k \in R V} D_{j k} \cdot \Delta E_{j k}(t-1) \\
& +\eta \cdot\left(n_{R I}\right)^{-1} \sum_{k \in R I} \Delta E_{j k}(t-1),
\end{aligned}
$$

where $n_{R V}$ is the number of evacuation individuals in the visual perception domain $R V$ of individual cell $j, n_{R I}$ is the number of evacuation individuals in the nonvisual perception domain $R I$ of individual cell $j, \eta$ is the degree to which the emotional difference of others in the nonvisual perception domain is received by individual $j$, and the value is not greater than the minimum distance weight in the visual perception domain, i.e., $0 \leq \eta \leq \min _{k \in R V} D_{j k}$.

Equation (5) calculates the average increment change in others' emotions perceived by pedestrians in the visual and nonvisual perception domains. It also indicates that the change in others' emotions is precisely related to distance. In the visual perception domain, the emotional difference in close distance has a great impact on pedestrian $j$, and it is the opposite in long distance. Moreover, in the nonvisual perception domain, the emotional difference perceived by pedestrian $j$ is weakened as a whole.

2.3. Update Mechanism of Emotional State. When the emotion accumulates to a certain extent, the state of pedestrians will change, and the emotion of pedestrians will be updated. The classical epidemic model SIS is a macrostatic mathematical model based on an ordinary differential equation, which can describe the contagion of emotion among groups but is not suitable for defining the state of free moving individuals in evacuation. In this paper, we modify it to reflect the microcontagion process of individual emotions. According to the SIS model, a pedestrian's tension can be defined as "susceptible emotional state" $(S)$ and "infectious emotional state" $(I)$. The transformation rules of the two emotional crowd states follow the SIS model. Fu et al. [25] proposed that the change of emotional threshold can lead to the dynamic change of evacuated pedestrians' status. Therefore, this paper defines the following. If the tension emotional intensity of "susceptible emotional state" $(S)$ is higher than the threshold $\lambda_{\text {emotion }}$, it turns into "infectious emotional state" $(I)$ with probability $\beta$, and $\beta$ is called the infection coefficient. If the tension emotional intensity of "infectious emotional state" (I) is lower than the threshold $\lambda_{\text {emotion, it turns into }}$ "susceptible emotional state" $(S)$ with probability $\gamma$, and $\gamma$ is called the calm coefficient. The state transition diagram is depicted in Figure 3.

Different evacuation individuals will adopt different evacuation strategies because of their different states. $\mathrm{Xu}$ et al. [16] analyzed the stampede accident video of "Love Parade" in Germany in 2010, observing that the crowd exhibited two different behavioral characteristics. One exhibited an active pedestrian (looking for the exit actively, accelerating forward, and obvious herd behavior), whereas the other exhibited an inactive pedestrian (trying to avoid the crowd, slow forward, and no obvious herd behavior). Accordingly, this paper associated pedestrian emotional intensity with pedestrian behavior: "susceptible emotional state" $(S)$ adopts "calm" strategy $B S$, speed is $v s$, and tends to choose the direction with low density $\rho$; "infectious emotional state" (I) adopts "impulsive" strategy $B I$, speed is $v i$, and tends to choose the direction with high density $\rho$. 


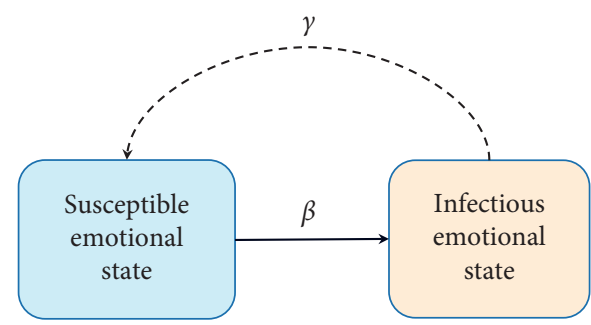

FIgURE 3: State transition diagram.

\section{Cellular Automata (CA) Simulation Model with Emotional Update}

This paper proposes an improved CA model with emotional contagion, entitled the CECA model. In this model, the cellular machine model is used to simulate and analyze the evacuation scenario in independent space. Pedestrians are randomly distributed in space, each occupying a cell. During an evacuation, pedestrians are affected by internal self emotion and others' external emotions, resulting in tension fluctuation. Each time, the pedestrian determines the next move strategy according to the transfer probability of the cell in each direction until the pedestrian reaches the exit and leaves the dangerous space safely.

Pedestrian intelligent behavior of following and memory timeliness can be indicated by dynamic FF of CA. In each time step, the pedestrian determines the direction according to the transition probability of the next moment. The transition probability is related to the distance from the neighborhood cell $C_{i}$ to the exit, whether it is an obstacle cell or a disaster cell. The population density of the forward direction is also a factor that determines the selection of target cells. In this model, the transition probability functions of "susceptible emotional state" $(S)$ and "infectious emotional state" $(I)$ groups are given.

When the emotional intensity is lower than $\lambda_{\text {emotion }}$, the "susceptible emotional state" group $S$ adopts the "calm" strategy $B S$, tending to choose the direction with low population density $\rho$. The speed is $v s_{j}$, and the maximum expected speed is $v_{j}^{\max }=0.8 \mathrm{~m} / \mathrm{s}$, moving one step per update. The target cells of "calm" pedestrian are eight cells around (blue cell), as shown in Figure 4(a). The target cells are assumed to be set to G. As depicted in Figure 4(b), the density range of "calm" pedestrian $j$ (red cell) is the density of close cells (gray cell) around neighborhood cell $i$ (blue cell). The transition probability of this type of pedestrian is calculated as follows:

$$
\left\{\begin{array}{l}
P_{j i}(t)=e^{N P_{B S}(t)} \cdot\left[\sum_{i \in G} e^{N P_{B S}(t)}\right]^{-1}, \\
N P_{B S}(t)=\left(k_{s} S_{j i}(t)+k_{\rho}(1-\rho(t))\right) \cdot n_{j i}(t) \cdot \alpha_{j i}(t) .
\end{array}\right.
$$

When the emotional intensity is higher than $\lambda_{\text {emotion}}$, the "infectious emotional state" group I adopts the "impulsive" strategy $B I$, tending to choose the direction with high population density $\rho$. The speed is $v i_{j}$, and the maximum expected speed is $v_{j}^{\max }=1.6 \mathrm{~m} / \mathrm{s}$, moving two steps per update. As depicted in Figure 5(a), the target cells of "impulsive" pedestrians are two step cells (blue cell) in each direction, assumed to be set to $G_{1}$. At this time, the moving speed is $v i_{j}=v_{j}^{\max }$. As depicted in Figure 5(b), the target cell with the highest transition probability among the eight cells in the neighborhood (blue cell) is selected if all the preferred eight cells are occupied. Then, the target cells are assumed to be set to $G_{2}$. At this time, the moving speed is $v i_{j}=0.8 \mathrm{~m} / \mathrm{s}$. The radius of the density range for "impulsive" pedestrians is two steps, as depicted in Figure 6. The transition probability of this type of pedestrian is calculated as follows:

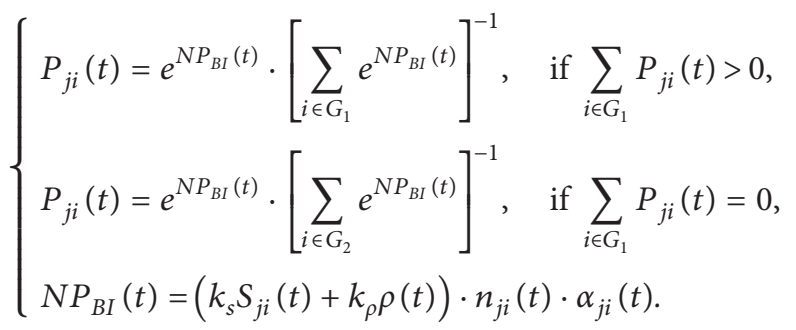

The parameters of the transition probability function are described below: $k_{s}$ is the influence coefficient of the distance to the exit, $k_{\rho}$ is the influence coefficient of the density, $n_{j i}$ is the state parameter of the neighborhood cell $i$ of the evacuation individual $j$ at time $t$ (if it has been occupied by the evacuation individual, it is equal to 0 ; otherwise, it is equal to 1), $\alpha_{j i}$ is whether the neighborhood cell $i$ of the evacuation individual $j$ at time $t$ is an obstacle (if it is an obstacle, it is equal to 0 ; otherwise, it is equal to 1$)$, and $\rho_{j i}(t)$ is the density around the neighborhood cell $i$ of the evacuation individual $j$ at time $t$, as shown in

$$
\rho_{j i}(t)=\frac{\sum_{i=1}^{\mathrm{num}}\left(n_{j i}(t)+\alpha_{j i}(t)\right)}{\text { num }},
$$

where num $=8$ because the Moore neighbor rule is followed.

$S_{j i}(t)$ is the shortest distance from the neighborhood cell $i$ of pedestrian $j$ to the exit at time $t$. Suppose that cell $\left(x_{i}, y_{i}\right)$ is the location coordinate of neighborhood cell $i$ of pedestrian $j$, there are $m$ effective exits in the evacuation space, and cell $\left(x_{e_{k}}, y_{e_{k}}\right)$ is the midpoint coordinate of each exit. According to the static floor field (SFF) rule of CA, $S_{j i}(t)$ is expressed as the following formula: 


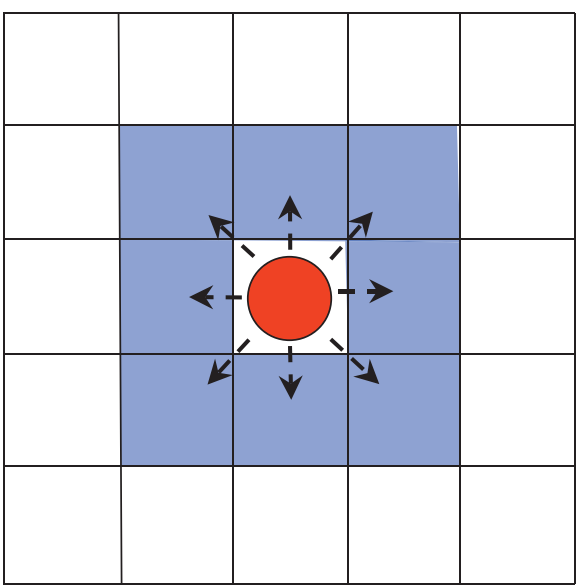

(a)

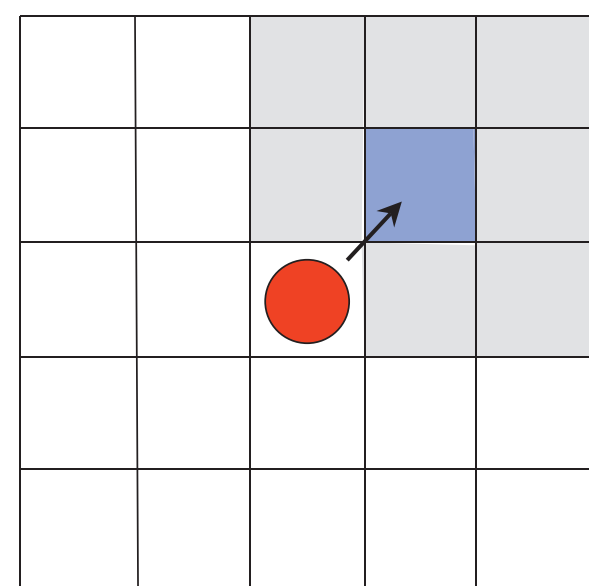

(b)

Figure 4: Density range of "calm" pedestrian.

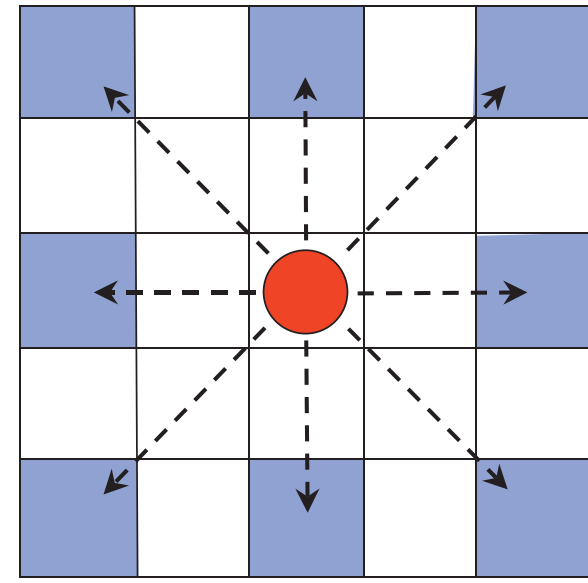

(a)

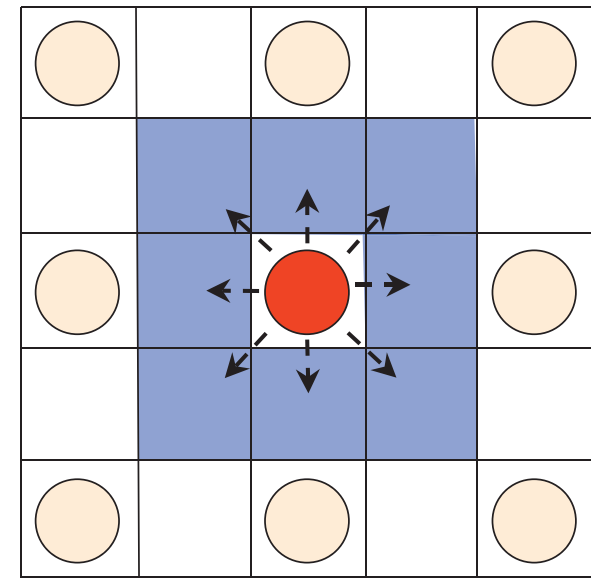

(b)

Figure 5: Possible target cells of "impulsive" pedestrian.

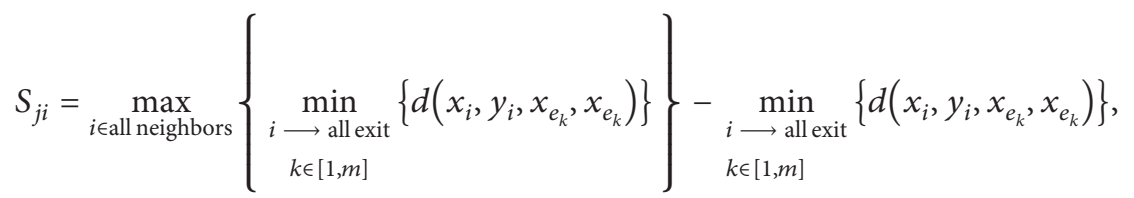

where $d\left(x_{i}, y_{i}, x_{e_{k}}, y_{e_{k}}\right)$ is the Euclidean distance from the neighborhood cell $i$ to the $k$ th exit.

There are two levels of progressive relationships in equation (9). First, the shortest distance between the eight cells in the neighborhood and all the effective exits is calculated. Second, the deviation between the shortest distance and its maximum is compared. If the deviation is 0 , then $S_{j i}(t)=0$; if the deviation is greater, then $S_{j i}(t)$ is greater, which indicates that the cell has the shortest distance from the exit, and the probability of the cell being selected is higher.
3.1. Update Rule. The tension emotional intensity of pedestrian fluctuates due to the contagion of others. Then, the evacuation strategy of each pedestrian is not single but dynamic. Considering that the emotion of pedestrians affects their movement in an emergency [16], the update process of the pedestrian state is as follows:

(1) The emotional state and evacuation space layouts are initialized.

(2) The SFF of each cell is calculated according to equation (9). 


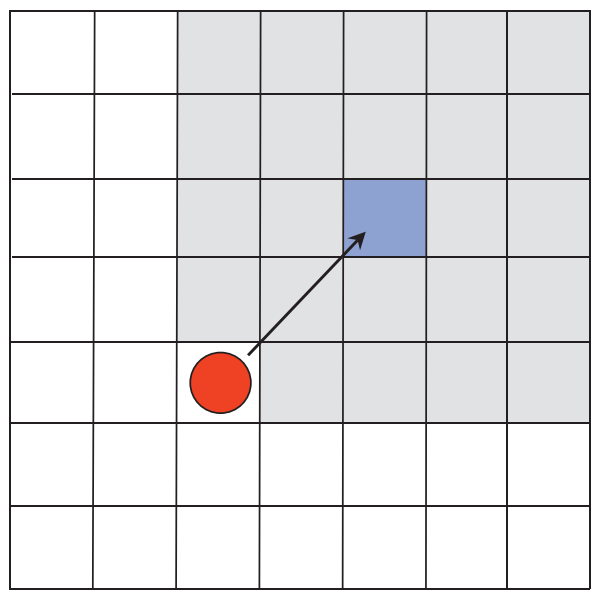

FIgURE 6: Density range of "impulsive" pedestrian.

(3) The crowd is randomly distributed in the evacuation space, and the pedestrian tension emotional intensity is initialized. If the emotional intensity is lower than the threshold $\lambda_{\text {emotion, }}$, the pedestrian position and emotional intensity will be recorded in the "susceptible emotional state" group $S$; otherwise, it will be recorded in the "infectious emotional state" group $I$.

(4) The emotional intensity is updated according to equations (1)-(5).

(5) The dynamic update of the evacuation population state is achieved. If the emotional intensity of "susceptible emotional state" group $S$ is higher than the threshold $\lambda_{\text {emotion }}$, it will change into "infectious emotional state" group $I$ with a probability of $\beta$. If the emotional intensity of "infectious emotional state" group $I$ is lower than the threshold $\lambda_{\text {emotion }}$, it will change into "susceptible emotional state" group $S$ with a probability of $\gamma$.

(6) The transition probability is calculated according to equations (6) and (7), and the target cell is selected.

(7) When multiple pedestrians select the same target cell, a pedestrian is randomly selected to move. This paper assumes that the probabilities of all pedestrians being selected are equal [22].

(8) When all pedestrians in the space have left, the simulation ends; otherwise, jump to (4).

(9) Every time the pedestrian moves, increment the pedometer by 1 until the end of the simulation.

\section{Experimental Simulation}

In this paper, the CECA model is compared with the classical CA model to verify its effectiveness. Then, the influence of emotional intensity threshold $\lambda_{\text {emotion }}$, infection coefficient $\beta$, calm coefficient $\gamma$, and perception radius $R$ on the evacuation efficiency of the system is analyzed.

4.1. Experiment 1. The following experiments are conducted to analyze the effectiveness of the improved model. The basic scene of [14] is used as the simulation experiment scene. The unit cell size is $c l \times c l$, and $c l=0.4 \mathrm{~m}$. The single exit room size is $18 \mathrm{cl} \times 14 \mathrm{cl}$, and the exit width is $e l_{1}=2 \mathrm{cl}$. When the initial state $T=0,150$ pedestrians are randomly distributed in the space, each occupying a cell. Each time step is $0.5 \mathrm{~s}$. The Moore neighbor rule is used when pedestrians move. In this paper, different parameters of the CECA model are used to perform multiple experiments, and the results are consistent. Only one set of experimental results is given here, and the corresponding parameters are $\lambda_{\text {emotion }}=0.5, \beta=0.5$, $\gamma=0.5$, and $\xi=0.0001$. Figures 7 and 8 are the density diagrams of the two models at different time steps.

As depicted in Figures 7 and 8, the pedestrian movement trend of the CECA model is consistent with that of the classical CA model over time. Pedestrians gather at the exit gradually, demonstrating the characteristics of arch flow at the exit-consistent with the characteristics of pedestrian movement flow. In the same time step, the pedestrian movement speed of the CECA model is faster than the CA model. The results demonstrate that the emotional infection and fluctuation of pedestrians in the model lead to dynamic change in pedestrian speed and reduce overall evacuation time.

The total evacuation time of the CECA model is compared with that of the classical CA model to verify further that the CECA model can reflect pedestrian movement characteristics. The results are depicted in Figure 9. It can be seen from Figure 9 that the trends of the two models are the same. When the number of people and the width of the exit are the same, the evacuation time of the CECA model is shorter because emotional infection is considered in the model and different evacuation strategies are adopted by pedestrian with different emotions.

4.2. Experiment 2. Several experimental studies were conducted to analyze the impact of emotional intensity threshold on the pedestrian evacuation process. The simulation scene is a single exit room. In the initial state, pedestrians are randomly distributed in the space. Each experiment consisted of 200 people with the same initial position and emotional value, and the system parameters are set to $\xi=0, \beta=1$, and $\gamma=1$. When the emotional threshold value is $\lambda_{\text {emotion }}=0.3,0.5$, and 0.7 , the simulation experiments are conducted on three parameters that reflect the dynamic characteristics of the group at each moment: the proportion of the group, the arch radius of the group at the exit, and the average moving speed in the system. The simulation program is executed 100 times in each case, and the average values are calculated. The results are depicted in Figures 10-12.

Based on Figure 10, when $\lambda_{\text {emotion }}=0.3$ and 0.5 , all pedestrian cells in the system are transformed into group $I$ at the end of the simulation. The smaller the threshold, the faster the conversion. When $\lambda_{\text {emotion }}=0.7$, all pedestrian cells are transformed into group $S$. The inflection point of the group state change can be approximated as $\lambda_{\text {emotion }}=0.5$.

Based on Figure 11, the total evacuation time with $\lambda_{\text {emotion }}=0.7$ is greater than that with $\lambda_{\text {emotion }}=0.3$ and 0.5 . 


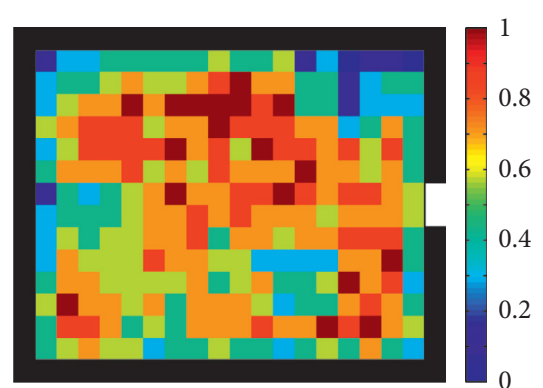

(a)

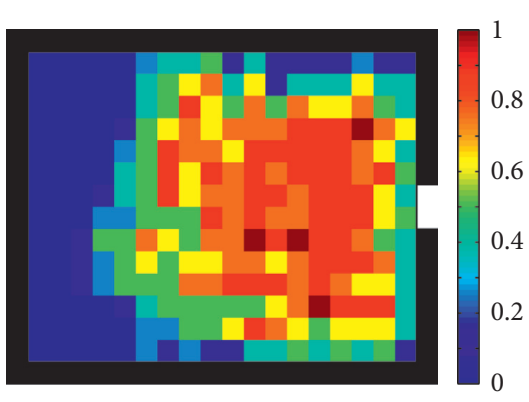

(b)

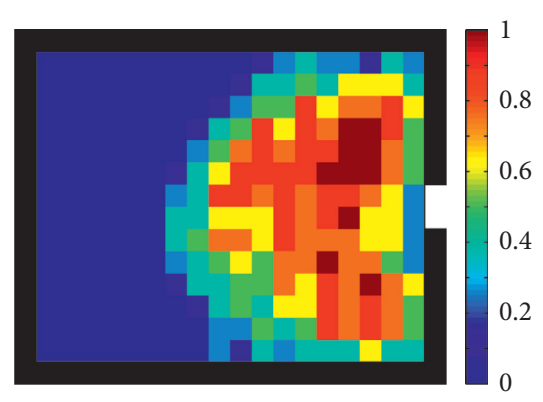

(c)

Figure 7: Pedestrian density of classical CA model at different times. (a) $T=0$. (b) $T=40$. (c) $T=80$.

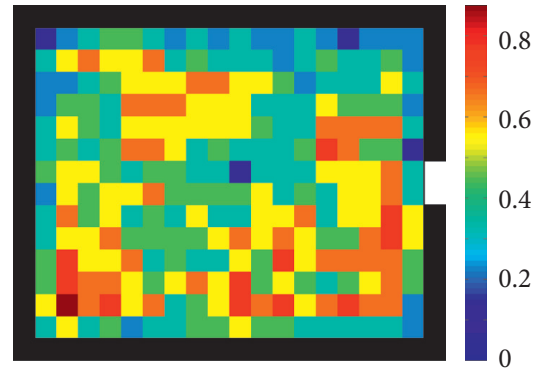

(a)

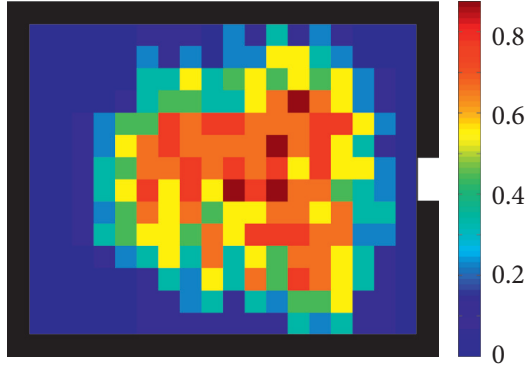

(b)

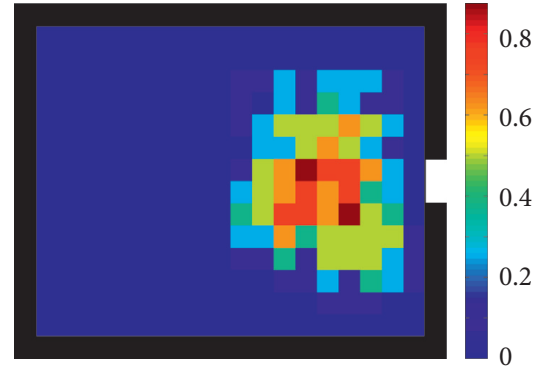

(c)

FIgure 8: Pedestrian density of the CECA model at different times. (a) $T=0$. (b) $T=40$. (c) $T=80$.

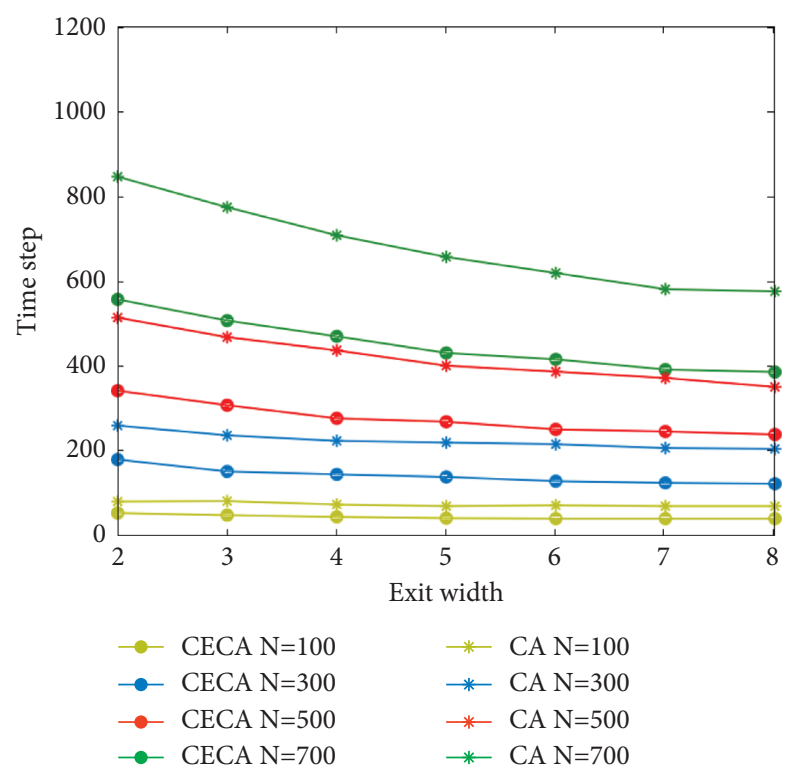

FIGURE 9: Comparison of the total evacuation time steps of the CECA model and classical CA model under different conditions.

The difference between $\lambda_{\text {emotion }}=0.3$ and $\lambda_{\text {emotion }}=0.5$ is minimal. When $T=0 \longrightarrow 30$, the exit arch radius is smaller; when $T=30 \longrightarrow 50$, the exit group radius of the three cases is close. This indicates that, when $\lambda_{\text {emotion }}=0.3$ and 0.5 , although the group moves quickly to the exit, it also accelerates the formation of aggregation at the exit, resulting in the phenomenon of queuing.

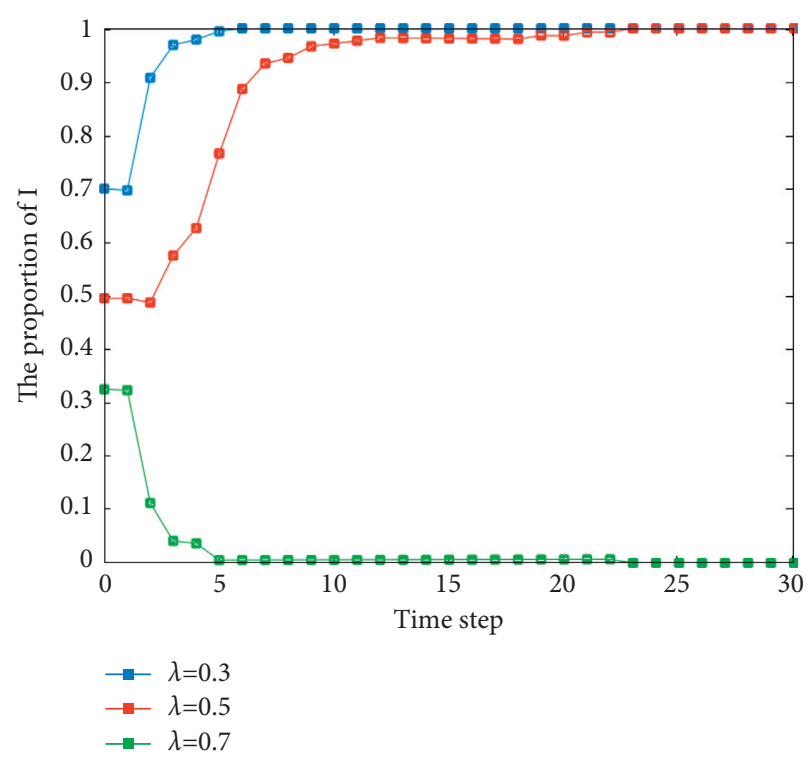

Figure 10: Proportion of group $I$ at each time.

The subsequent analysis is performed for $T=50 \longrightarrow 70$. Based on Figure 10, when $\lambda_{\text {emotion }}=0.3$ and 0.5 , the site is full of group $I$, and when $\lambda_{\text {emotion }}=0.7$, the site is full of group $S$. At this stage, the decrease in population density leads to an increase in opportunities for groups to choose to move; group $I$ illustrates the characteristics of faster evacuation than group $S$. 


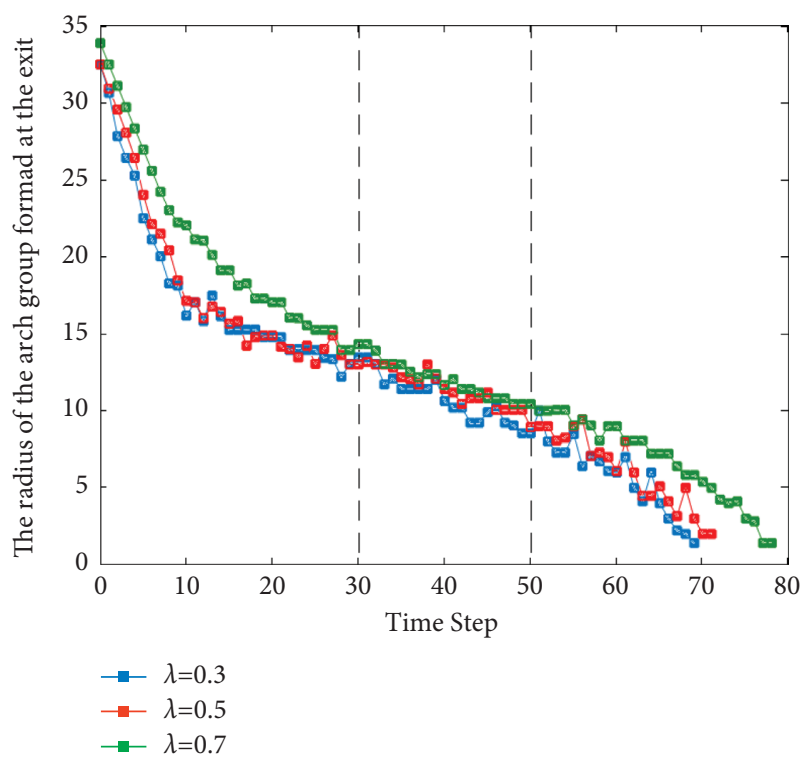

FIGURE 11: Radius of the arch group formed at the exit at each time.

Figure 12 illustrates the average evacuation speed of the system at each time. When $T=0 \longrightarrow 30$, the speed of the three cases decreased rapidly. When $T=30 \longrightarrow 50$, the average speed fluctuates in a small range because the radius of the arch group formed at the exit in the three cases are almost the same in Figure 11. At this time, the exit is more crowded, resulting in some evacuation individuals unable to move, so the average moving speed is small. When $T=50 \longrightarrow 70$, the average moving speed curve of the system rises, indicating that the average speed of the entire system first decreases and then increases. Simultaneously, the average moving speed of the system at $\lambda_{\text {emotion }}=0.3$ and 0.5 is higher than that at $\lambda_{\text {emotion }}=0.7$.

4.3. Experiment 3. Infection coefficient $\beta$ and calm coefficient $\gamma$ are essential factors of individual emotion driving behavior change during an evacuation. Based on the analysis of the previous experiment, this simulation experiment considers two situations of emotional threshold $\lambda_{\text {emotion }}=0.5$ and $\lambda_{\text {emotion }}=0.6$, to explore the group state of the system when the infection coefficient $\beta$ and calm coefficient $\gamma$ change independently. Consider the same group of 200 people with a fixed initial emotional value and fixed initial position. Four scenario experiments with different parameters were designed: Scenario 1: $\lambda_{\text {emotion }}=0.5, \quad \gamma=0.1, \quad$ and $\beta=0.1,0.3,0.5,0.7$, and 0.9; Scenario $2: \quad \lambda_{\text {emotion }}=0.6, \quad \gamma=0.1, \quad$ and $\beta=0.1,0.3$, $0.5,0.7$, and 0.9; Scenario 3: $\lambda_{\text {emotion }}=0.5, \beta=0.1$, and $\gamma=0.1,0.3,0.5,0.7$, and $0.9 ; \quad$ Scenario $4: \quad \lambda_{\text {emotion }}=0.6$, $\beta=0.1$, and $\gamma=0.1,0.3,0.5,0.7$, and 0.9. The simulation program is executed 100 times in each case, and the average values are calculated. The simulation results are depicted in Figure 13.

In Scenario 1, the higher the infection coefficient $\beta$, the faster the proportion of group $I$ increases from 0.5 to 1 . In

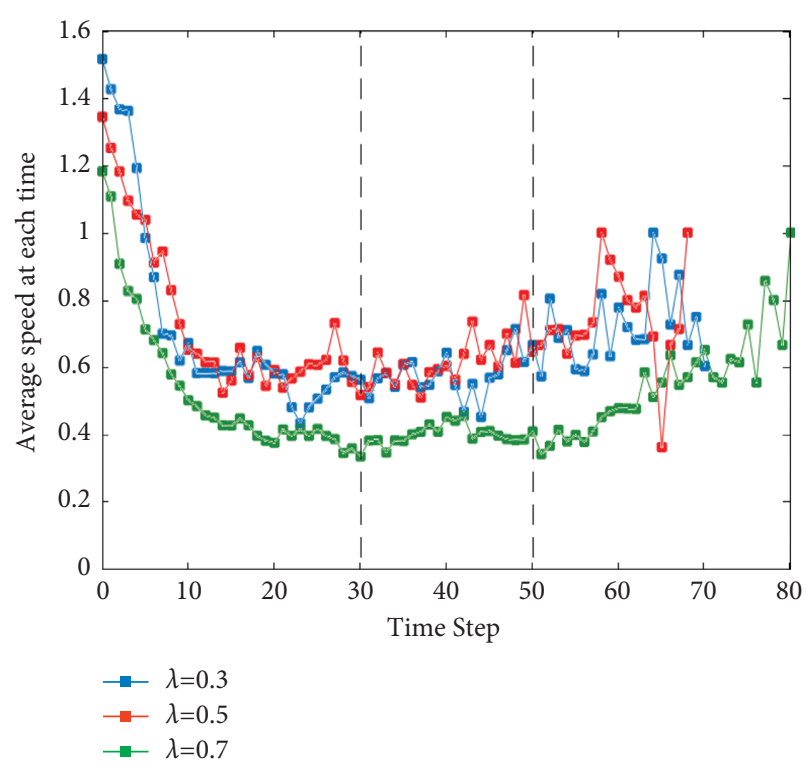

Figure 12: Average speed at each time.

Scenario 2, regardless of the infection coefficient $\beta$, the number of group $I$ gradually decreases. Moreover, the value of $\beta$ has no significant effect on the reduction of group $I$. In Scenario 3, the proportion of group $I$ tends to 1 eventually. In the early stage of an evacuation, the larger the calm coefficient $\gamma$, the more the proportion of group I decreases, which means that the larger the proportion of group $S$ is.

However, the proportion of group $I$ trended upward after a small decline. It oscillates to 1 , especially after $T=40$. During this period, $\gamma$ had no significant effect on the proportion of $I$. In Scenario 4, the pedestrian cells are all group $S$. The larger the calm coefficient $\gamma$, the faster the transition to group $S$.

4.4. Experiment 4. Based on the previous analysis, emotional threshold $\lambda_{\text {emotion }}$, infection coefficient $\beta$, and calm coefficient $\gamma$ are the three critical parameters that affect the individual evacuation state. This paper analyzes the impact of these three factors on the overall evacuation time by conducting simulation experiments on the evacuation time when $\lambda_{\text {emotion }}=0.2,0.4,0.6$, and 0.8 , the number of people $N=100$ and 200, and the infection coefficient $\beta$ and calm coefficient $\gamma$ change simultaneously. The simulation program is executed 100 times in each case, and the average values are calculated.

The experimental results are depicted in Figures 14 and 15. Each figure represents the three-dimensional aerial view of the total evacuation time at different $\lambda_{\text {emotion. The hori- }}$ zontal axis is the calm coefficient $\gamma$, and the vertical axis is the infection coefficient $\beta$. Different colors indicate the values of the evacuation time, which tends to be higher in red and lower in blue.

As depicted in Figures 14 and 15, the total evacuation time of $\lambda_{\text {emotion }}=0.2$ and $\lambda_{\text {emotion }}=0.4$ are lower than those of $\lambda_{\text {emotion }}=0.6$ and $\lambda_{\text {emotion }}=0.8$. When the emotional thresholds are $\lambda_{\text {emotion }}=0.2$ and $\lambda_{\text {emotion }}=0.4$, the larger the 


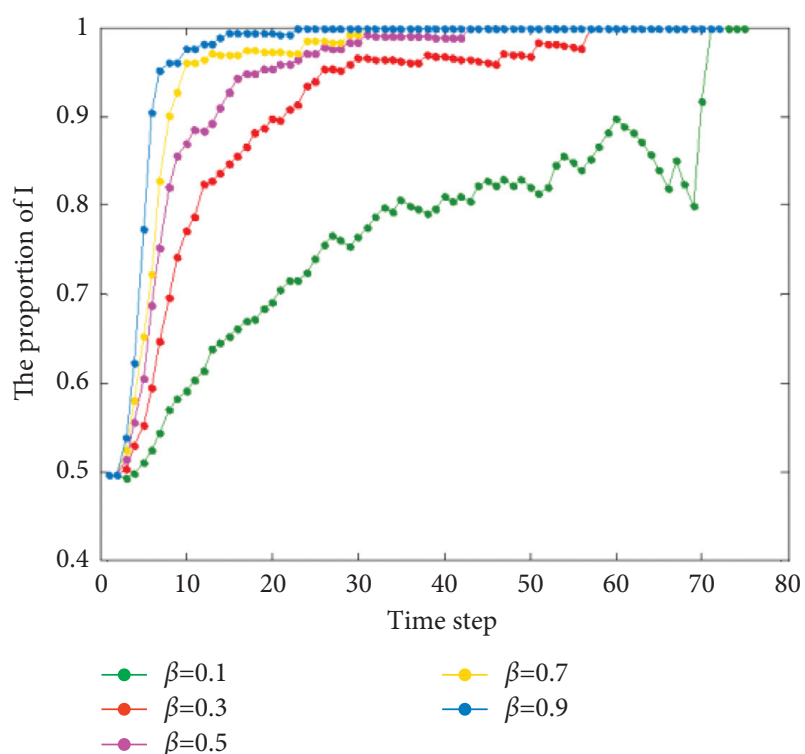

(a)

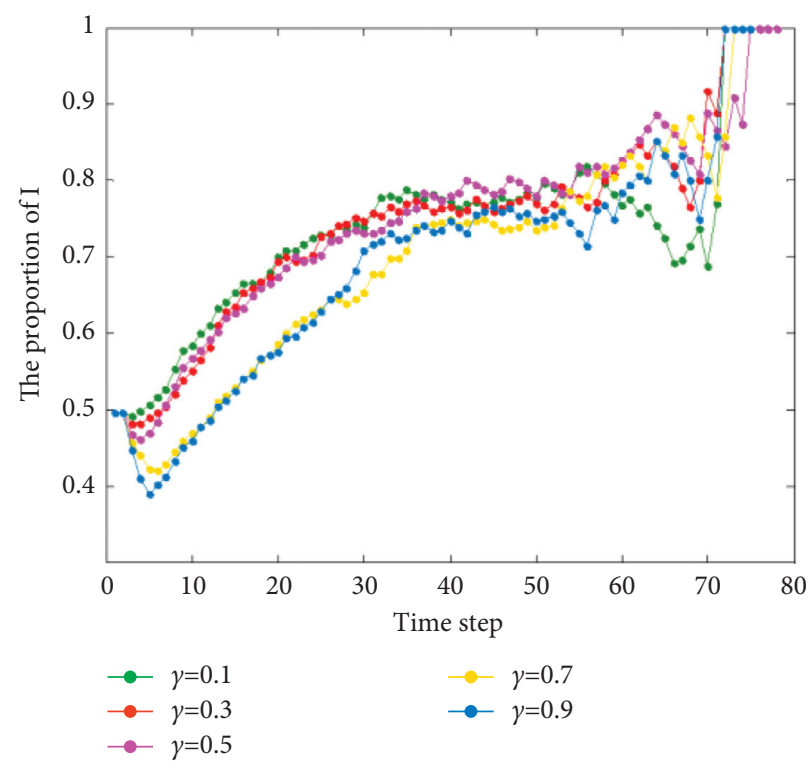

(c)

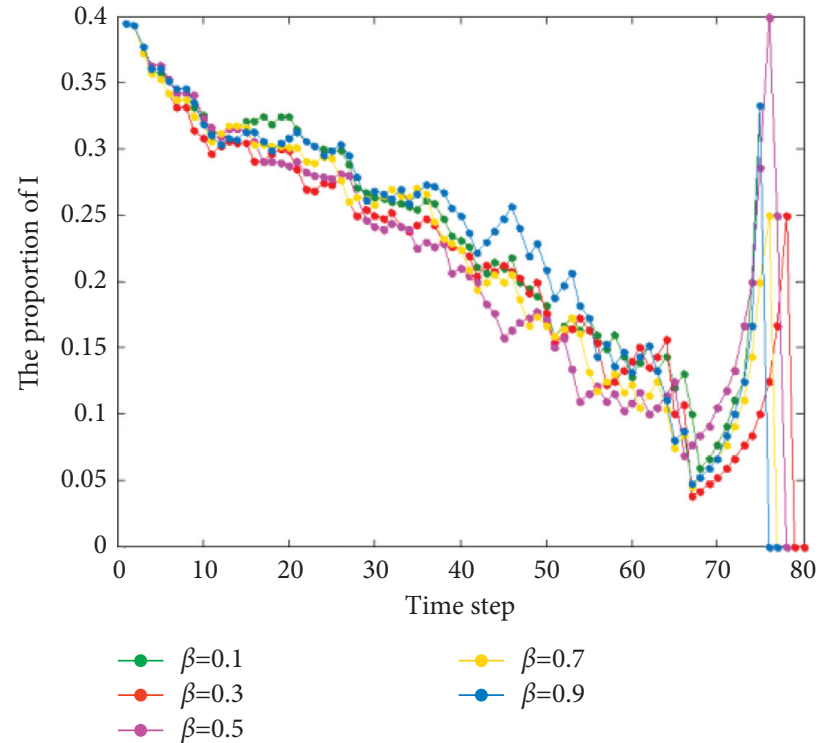

(b)

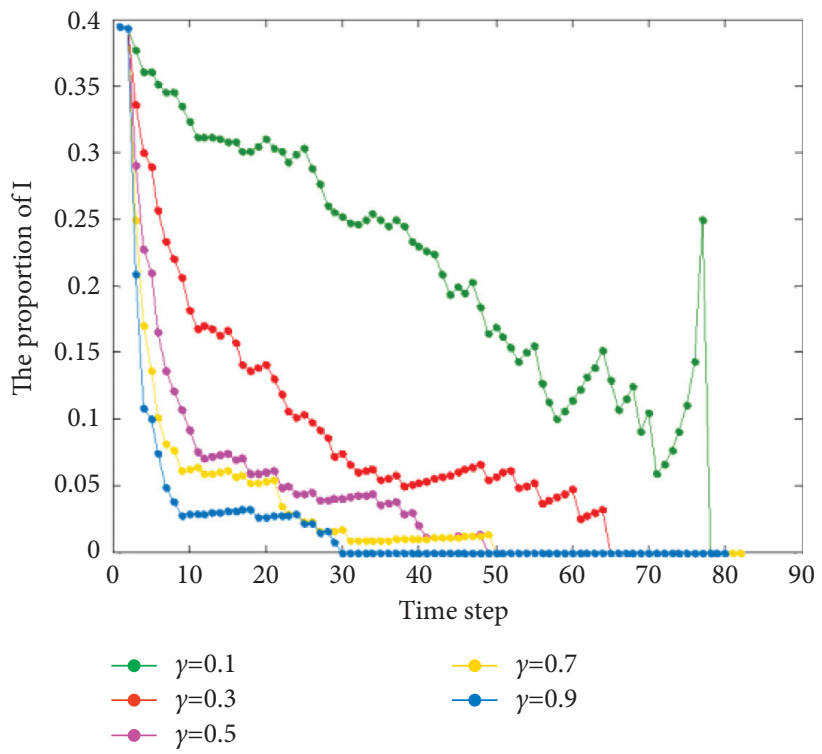

(d)

FiguRe 13: Proportion of $I$ under different thresholds and state transition parameters. (a) Scenario $1\left(\lambda_{\text {emotion }}=0.5\right.$ and $\left.\gamma=0.1\right)$. (b) Scenario $2\left(\lambda_{\text {emotion }}=0.6\right.$ and $\left.\gamma=0.1\right)$. (c) Scenario $3\left(\lambda_{\text {emotion }}=0.5\right.$ and $\left.\beta=0.1\right)$. (d) Scenario $4\left(\lambda_{\text {emotion }}=0.6\right.$ and $\left.\beta=0.1\right)$.

infection coefficient $\beta$, the smaller the total evacuation time. This result is more apparent when $N=200$ in Figure 15. In contrast, when the infection coefficient $\beta$ tends toward 0 , the total evacuation time increases. When the emotional threshold is $\lambda_{\text {emotion }}=0.6$ and $\lambda_{\text {emotion }}=0.8$, the larger the calm coefficient $\gamma$, the larger the total evacuation time.

Based on Figures 14 and 15, when $\gamma>0.5$, the total evacuation time is relatively large. In this case, when $\lambda_{\text {emotion }}>0.5$, the proportion of $S$ in the initial group is larger. In addition, $\gamma>0.5$ indicates that the probability of transition from $I$ to $S$ is higher; combined with the analysis of Experiment 3, the larger the $\gamma$ value, the faster the transition to group $S$, which will lead to a longer evacuation time.
4.5. Experiment 5. The emotion of evacuation individuals will fluctuate dynamically during an evacuation due to the perception domain. In the improved model, the emotional perception area of evacuation individuals is a circle with radius $R$. This experiment analyzes the influence of perception domain radius $R$ on the total evacuation time. Set a single exit room of size $12 \mathrm{~m} \times 12 \mathrm{~m}$ as the simulation scene. The unit cell size is $c l \times c l, c l=0.4 \mathrm{~m}$, exit width is $e l_{1}=3 c l$, and 200 people were randomly distributed in the space and assigned a random initial emotional values. In order to facilitate the analysis of the influence of perception radius $R$, set the alternative parameter $n n=(R / c l)$; then, $n n=1,2,3,4$, and 5 . 

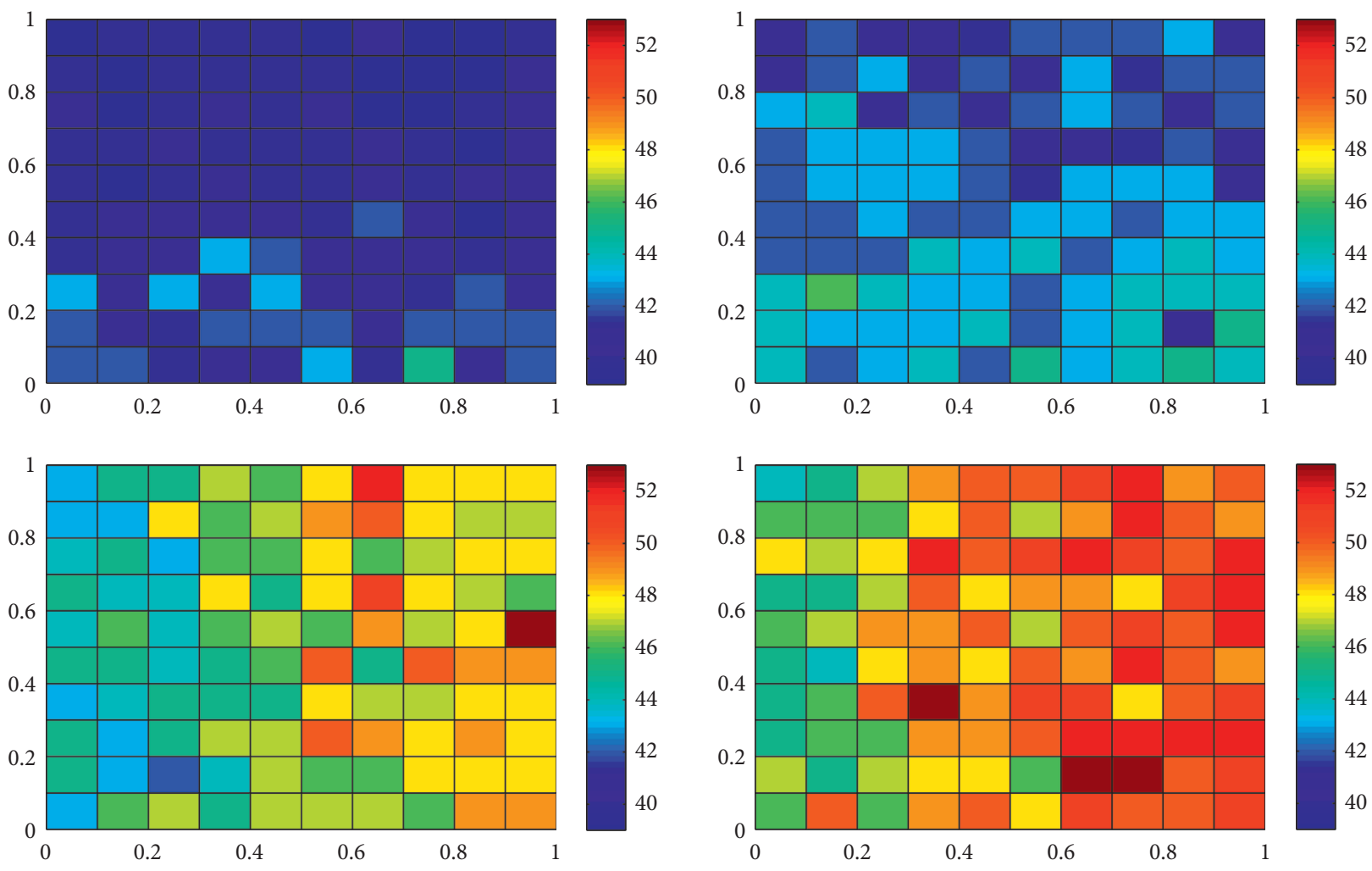

Figure 14: Total evacuation time $(N=100)$.

Three different scenarios with different parameters are designed for the simulation experiment: Scenario 1: $\beta=0.33$ and $\gamma=0.67$; Scenario $2: \beta=0.5$ and $\gamma=0.5$; Scenario 3: $\beta=0.67$ and $\gamma=0.33$. The influence of different perception radius $n n$ on the total evacuation time under three scenarios with emotional thresholds of $\lambda_{\text {emotion }}=0.2,0.4,0.6,0.8$ is analyzed. Each experiment runs 100 times, and the average values of the total evacuation time are calculated. The simulation results are depicted in Figure 16.

Based on Figure 16, there is no difference in the total evacuation time between Scenario 1 and Scenario 3 when $\lambda_{\text {emotion }}=0.2$. In Scenario 2, the total evacuation time of the perception radius $n n=4$ and 5 is smaller. For all three scenarios, when $\lambda_{\text {emotion }}=0.4$, the total evacuation time of perception radius $n n=4$ and 5 is less than $n n=1,2$, and 3 . When $\lambda_{\text {emotion }}=0.6$ and $\lambda_{\text {emotion }}=0.8$, the three scenarios show that the minimum total evacuation time appears at $n n=1$, and the larger the perception domain radius, the larger the total evacuation time. Although the total evacuation time decreases at $n n=4$ when $\lambda_{\text {emotion }}=0.6$ in Scenario 3 , it is also higher than that at $n n=1$. Therefore, it can be concluded that when $\lambda_{\text {emotion }} \leq 0.5$, the larger the perception radius $n n$, the shorter the total evacuation time, and when $\lambda_{\text {emotion }}>0.5$, the opposite occurs. This paper verifies this conclusion by conducting simulation experiments on the same three scenarios with initial numbers of 300 and 400, and the conclusions are consistent.
4.6. Experiment 6. In the previous experiments, the initial emotion of evacuation individuals was randomly given in the range of emotional intensity, and the effects of different system parameters on evacuation efficiency were analyzed. However, when an emergency event occurs, it will induce the overall emotional tension of pedestrians in the system. The purpose of this experiment is to use the improved model to study the changes in the group state under different emotions. The experimental scene is the same as that of the previous experiment. Combined with the previous experiments, the system parameters are set to be favorable for evacuation time: $\lambda_{\text {emotion }}=0.4, \beta=0.6, \gamma=0.5, n n=5$, and $\xi=0.0001$.

In the initial state, 200 individuals were randomly distributed in the space. Three different scenarios were designed: Scenario 1: emotions are randomly assigned in the range of $[0,1]$; Scenario 2: emotions are randomly assigned in the range $[0.2,1]$; Scenario 3: emotions are randomly assigned in the range $[0.4,1]$. The positions of two types of state groups in the evacuation site are depicted at specific time $T$ in Figure 17. The evacuation pedestrians of group $S$ (blue), group $I$ (red), and exit (green) are illustrated.

As depicted in Figure 17, in Scenario 1, the overall emotion of pedestrians is in the normal range. At $T=0$, the number of groups $I$ and $S$ is equal, and when $T=5$, the number of group $I$ is significantly more than that of group $S$. In Scenario 2, the overall emotion is slightly tense. At $T=0$, the number of group $I$ is greater than that of group $S$. When 

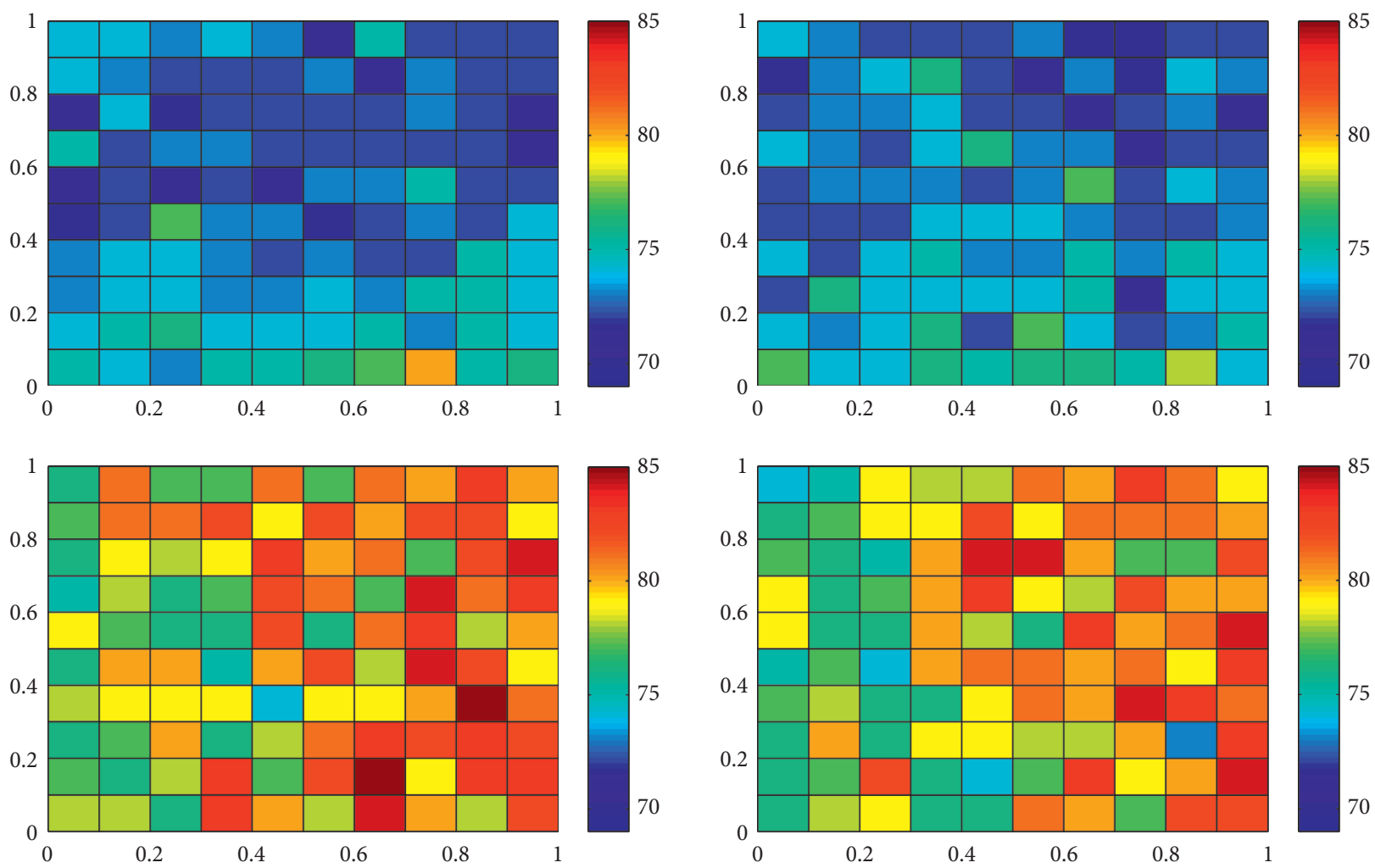

Figure 15: Total evacuation time $(N=200)$.
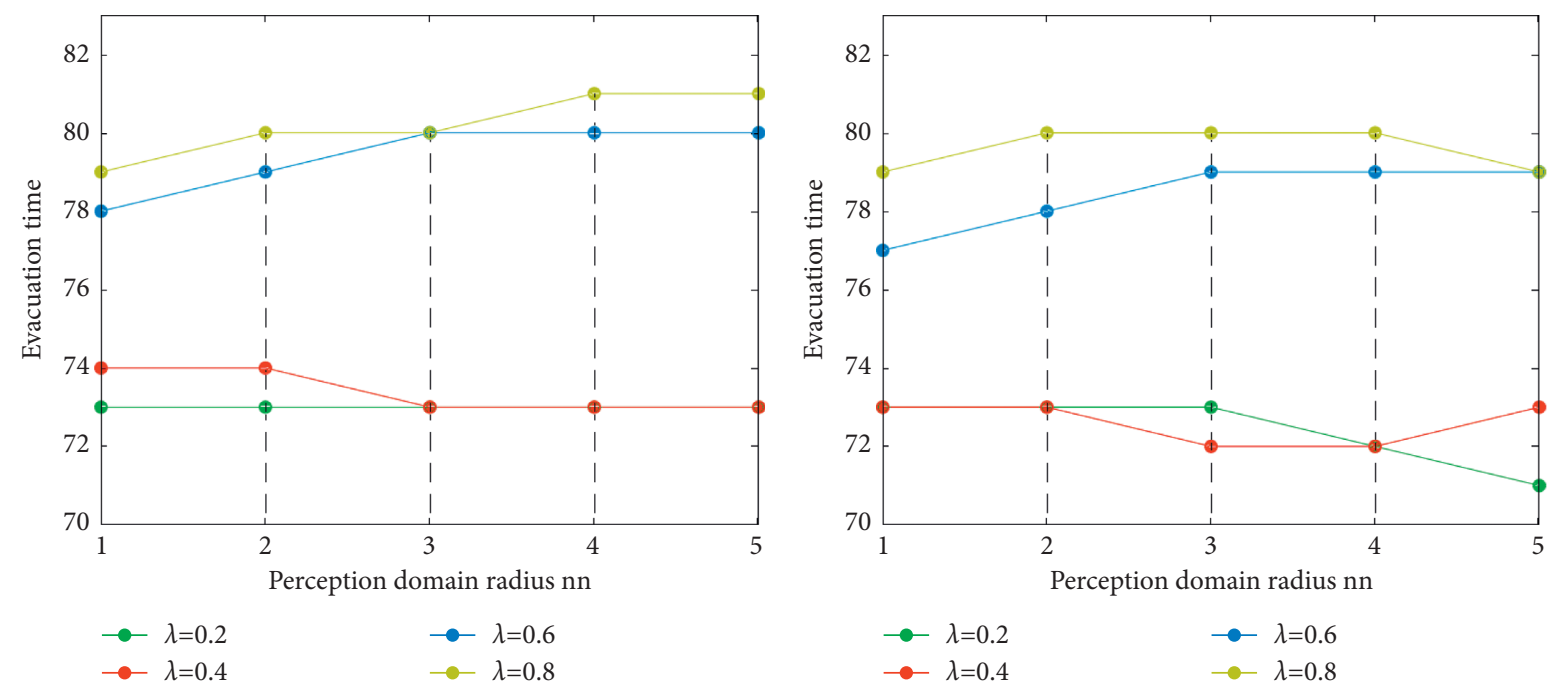

$\longrightarrow \lambda=0.2$
$\longrightarrow \lambda=0.4$

$\rightarrow \lambda=0.6$

(a)

(b)

Figure 16: Continued. 


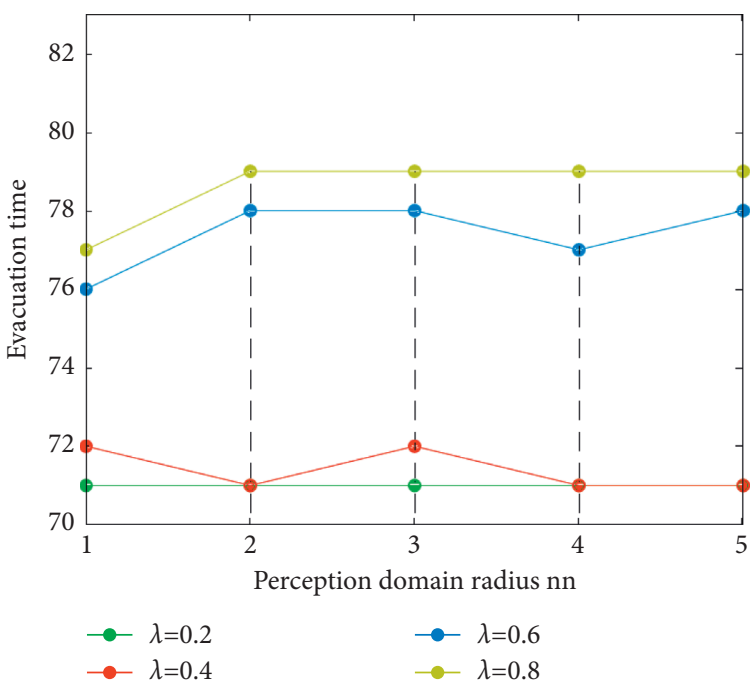

(c)

FiguRE 16: Total evacuation time of different perception radii under different thresholds and state transition parameters. (a) Scenario 1 $(\beta=0.33$ and $\gamma=0.67)$. (b) Scenario $2(\beta=0.5$ and $\gamma=0.5)$. (c) Scenario $3(\beta=0.67$ and $\gamma=0.33)$.

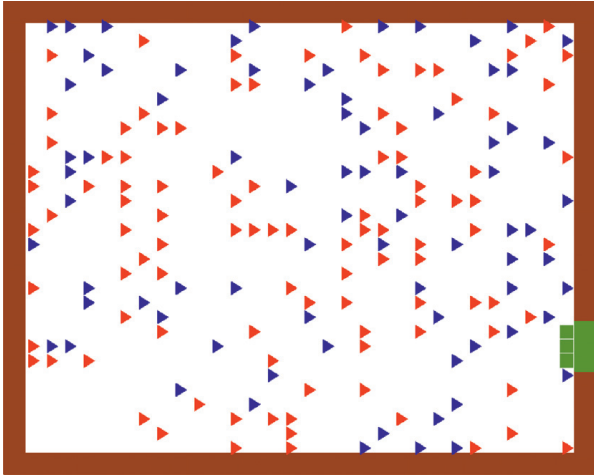

(a)

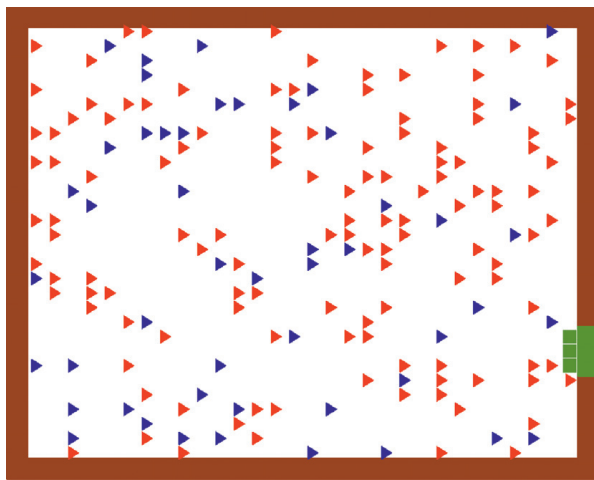

(c)

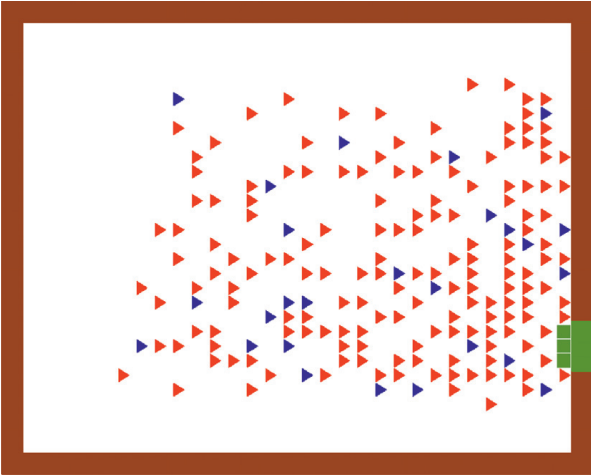

(b)

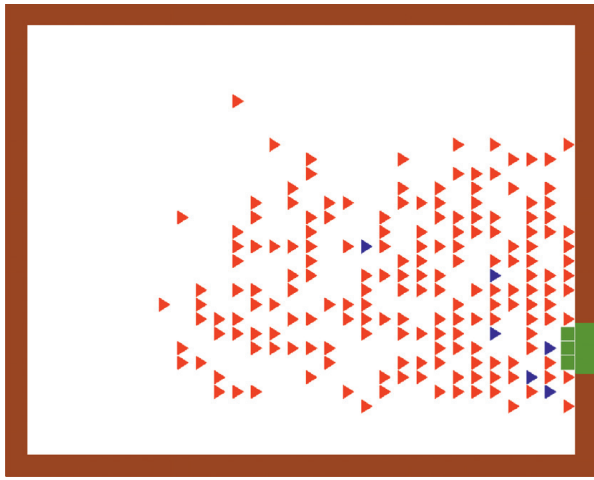

(d)

Figure 17: Continued. 


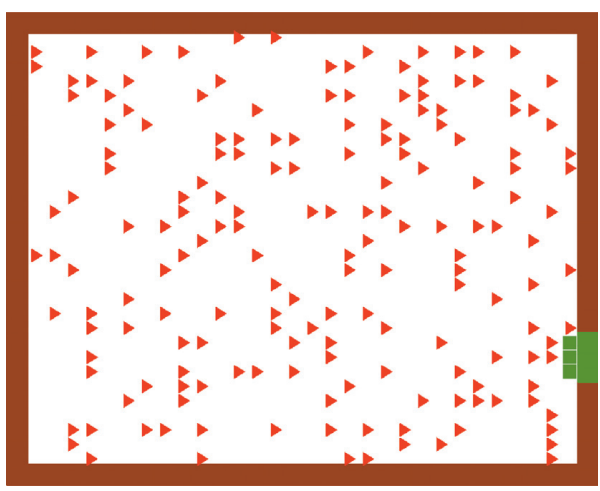

(e)

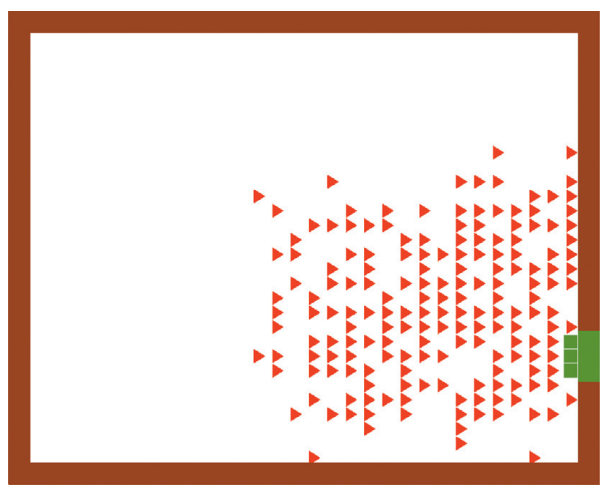

(f)

Figure 17: Population distribution of evacuation individuals at $T=0$ and $T=5$ under different emotions. (a) Scenario 1 ( $T=0$ ). (b) Scenario $1(T=5)$. (c) Scenario $2(T=0)$. (d) Scenario $2(T=5)$. (e) Scenario $3(T=0)$. (f) Scenario $3(T=5)$.

$T=5$, most of the pedestrians turn into group $I$ and adopt the "impulsive" strategy after a short evacuation, demonstrating the phenomenon of a crowd closing to the exit. In Scenario 3, an extreme scenario is simulated, and the overall pedestrian emotion is more tense. At $T=0$, pedestrians are in state $I$, while when $T=5$, the entire crowd gathers at the exit rapidly.

It can be seen that, in Scenario 2 and Scenario 3, the overall pedestrian emotion is tense, which shows that pedestrians gather rapidly. Especially, in Scenario 3, dangerous situations such as crowding, pushing, and even trampling are prone to occur, which will affect the evacuation efficiency. Therefore, a management strategy to improve evacuation efficiency should be adopted during the evacuation. Simultaneously, measures should be taken to appease the excessive tension and panic of pedestrians.

\section{Conclusions}

Because research on dynamic evacuation behavior considering emotional factors is currently limited and the model of dynamic update of emotion and behavior decision-making remains to be studied, this paper proposes an improved pedestrian evacuation CA model, entitled the CECA model. The improved model can effectively achieve the dynamic evolution of pedestrian evacuation considering emotional infection by defining the direction perception domain and dynamic emotional perception. Compared with the classical CA pedestrian evacuation model, the CECA model can represent crowd conformity and exit arch characteristics and can simulate the pedestrian evacuation process more efficiently. Through the simulation analysis of the model parameters, the following conclusions are obtained:

(1) The dynamic change in the group is affected by the emotional threshold. When $\lambda_{\text {emotion }} \leq 0.5$, all pedestrian cells are transformed into group $I$, and when $\lambda_{\text {emotion }}>0.5$, all pedestrian cells are transformed into group $S$. Different groups adopt different evacuation strategies. For evacuation managers, evacuation management measures can be used to maintain $\lambda_{\text {emotion }}$ lower than 0.4 as far as possible, which is conducive to a rapid evacuation.
(2) The effects of the infection coefficient $\beta$ and calm coefficient $\gamma$ differ under different emotional thresholds. When $\lambda_{\text {emotion }} \leq 0.5$, the influence of the infection coefficient $\beta$ is higher. The higher the value of $\beta$, the faster the group $S$ transforms into group $I$. Finally, the entire group $I$ is formed, resulting in less evacuation time. When $\lambda_{\text {emotion }}>0.5$, the influence of the infection coefficient $\gamma$ is higher. The higher the value of $\gamma$, the faster the group $I$ transforms into group $S$. Finally, the entire group $S$ is formed, and the total evacuation time is relatively high. This conclusion provides an essential basis for evacuation managers to understand the factors of group state change to control evacuations effectively.

(3) The effect of perception radius on total evacuation time was also related to the emotional threshold. When $\lambda_{\text {emotion }} \leq 0.5$, the larger the perception radius of the evacuation individual, the higher the average emotional value of the system and the more conducive it is for the evacuation individual to change into group $I$ and leave the site quickly. When $\lambda_{\text {emotion }}>0.5$, the larger the perception radius of individual evacuation, the lower the average emotional value of the system and the easier it is for the evacuation individual to change into group $S$, resulting in a longer evacuation time.

(4) Due to the "conformity" of the evacuated individuals, when the general emotional concentration of the surrounding people is high, the individuals must perceive and enhance the possibility of state transition. At this time, individuals generally adopt the "impatient" strategy. Although the action is to speed up the evacuation, the overall emotional tension will also lead to the crowd quickly gathering at the exit. This phenomenon leads easily to large crowds, stampedes, and other dangerous events, so it should be avoided as much as possible.

These conclusions are helpful for evacuation managers to understand the change factors of group behavior state and strategy of evacuation individuals. Managers can promote $\lambda_{\text {emotion }} \leq 0.5$ and increase the infection coefficient $\beta$ through 
an external management strategy to increase the probability of transition from group $S$ to group $I$. Furthermore, they can guide the evacuation individuals to expand their vision, observe distant areas, and engage in positive action strategies. If $\lambda_{\text {emotion }}>0.5$, managers can use management strategies to suppress the calm coefficient $\gamma$ and control the probability of the transition from group $I$ to group $S$. Simultaneously, they can guide the evacuation individuals to pay attention to the emotion or behavior strategy of others nearby, which is more conducive to changing personal emotion and state, to achieve the purpose of evacuation as soon as possible. In addition, some measures should be taken to avoid excessive group tension and panic. The improved model is more consistent with the actual evacuation, but there are still some aspects to be improved in future research. The simulation results require the support of empirical data. Empirical experiments will be conducted to verify the significance of the conclusions of this paper in the future.

\section{Data Availability}

The data used to support the findings of this study are available from the corresponding author upon request.

\section{Conflicts of Interest}

The authors declare that there are no conflicts of interest regarding the publication of this paper.

\section{Acknowledgments}

This study was supported by the Liaoning Natural Science Foundation of China (Grant no. 2019-ZD-0558).

\section{References}

[1] X. Li, F. Guo, H. Kuang, Z. Geng, and Y. Fan, “An extended cost potential field cellular automaton model for pedestrian evacuation considering the restriction of visual field," Physica A: Statistical Mechanics and Its Applications, vol. 515, pp. 47-56, 2019.

[2] H. Zhang, H. Liu, X. Qin, and B. Liu, "Modified two-layer social force model for emergency earthquake evacuation," Physica A: Statistical Mechanics and Its Applications, vol. 492, pp. 1107-1119, 2018.

[3] X. Xu and W. Song, "Staircase evacuation modeling and its comparison with an egress drill," Building and Environment, vol. 44, no. 5, pp. 1039-1046, 2009.

[4] Y.-F. Wei, W. Shi, and T. Song, "Approach to effect of obstacle on pedestrian evacuation with a small-grid lattice gas model," Procedia Engineering, vol. 31, pp. 1077-1082, 2012.

[5] S. J. Gress and D. A. Simone, "Evacuation characteristics of visually impaired people-a qualitative and quantitative study," Fire \& Materials, vol. 39, no. 4, 2013.

[6] V. J. Blue, M. J. Embrechts, and J. L. Adler, "Cellular automata modeling of pedestrian movements," in Proceedings of the 1997 IEEE International Conference on Systems, Man, and Cybernetics, Orlando, FL, USA, 1997.

[7] C. Burstedde, K. Klauck, A. Schadschneider, and J. Zittartz, "Simulation of pedestrian dynamics using a two-dimensional cellular automaton," Physica A, vol. 295, no. 3-4, pp. 507-525, 2001.
[8] A. Schadschneider, "Traffic flow: a statistical physics point of view," Physica A, vol. 313, no. 1-2, pp. 153-187, 2002.

[9] W. Fang, L. Yang, and W. Fan, "Simulation of bi-direction pedestrian movement using a cellular automata model," Physica A, vol. 321, no. 3-4, pp. 633-640, 2003.

[10] L. Yang, J. Li, and S. Liu, "Simulation of pedestrian counterflow with right-moving preference," Physica A, vol. 387, no. 15, pp. 3281-3289, 2008.

[11] H. Yue, H. Hao, X. Chen, and C. Shao, "Simulation of pedestrian flow on square lattice based on cellular automata model," Physica A: Statistical Mechanics and Its Applications, vol. 384, no. 2, pp. 567-588, 2007.

[12] J. Ji, L. Lu, Z. Jin, S. Wei, and L. Ni, "A cellular automata model for high-density crowd evacuation using triangle grids," Physica A: Statistical Mechanics and Its Applications, vol. 509, pp. 1034-1045, 2018.

[13] Y. Zheng, X. Li, N. Zhu, B. Jia, and R. Jiang, "Evacuation dynamics with smoking diffusion in three dimension based on an extended floor-field model," Physica A: Statistical Mechanics and Its Applications, vol. 507, pp. 414-426, 2018.

[14] M. Shi, E. W. M. Lee, and Y. Ma, "A dynamic impatiencedetermined cellular automata model for evacuation dynamics," Simulation Modelling Practice and Theory, vol. 94, pp. 367-378, 2019.

[15] Z. Geng, X. Li, H. Kuang, X. Bai, and Y. Fan, "Effect of uncertain information on pedestrian dynamics under adverse sight conditions," Physica A: Statistical Mechanics and Its Applications, vol. 521, pp. 681-691, 2019.

[16] T. Xu, D. Shi, J. Chen, T. Li, P. Lin, and J. Ma, "Dynamics of emotional contagion in dense pedestrian crowds," Physics Letters A, vol. 384, Article ID 126080, 2019.

[17] P. T. Costa and R. R. McCrae, "Personality in adulthood: a five-factor theory perspective," International and Cultural Psychology, pp. 303-322, Springer, Berlin, Germany, 2002.

[18] D. Helbing, I. Farkas, and T. Vicsek, "Simulating dynamical features of escape panic," Nature, vol. 407, no. 6803, pp. 487-490, 2000.

[19] H. F. David, P. F. Pamela, O. M. Mireia, and J. Richard, "The influence of emotional state on risk perception in pedestrians: a psychophysiological approach," Safety Science, vol. 130, Article ID 104857, 2020.

[20] L. Fu, W. Song, W. Lv, X. Liu, and S. Lo, "Multi-grid simulation of counter flow pedestrian dynamics with emotion propagation," Simulation Modelling Practice and Theory, vol. 60, pp. 1-14, 2016.

[21] C. Chen, H. Sun, P. Lei, D. Zhao, and C. Shi, "An extended model for crowd evacuation considering pedestrian panic in artificial attack," Physica A, vol. 571, Article ID 125833, 2021.

[22] L. Zheng, X. Peng, L. Wang, and D. Sun, "Simulation of pedestrian evacuation considering emergency spread and pedestrian panic," Physica A: Statistical Mechanics and Its Applications, vol. 522, pp. 167-181, 2019.

[23] X. Li, F. Guo, H. Kuang, and H. Zhou, "Effect of psychological tension on pedestrian counter flow via an extended cost potential field cellular automaton model," Physica A: Statistical Mechanics and Its Applications, vol. 487, pp. 47-57, 2017.

[24] C. Cocking, J. Drury, and S. Reicher, "The psychology of crowd behaviour in emergency evacuations: results from two interview studies and implications for the fire and rescue services," Irish Journal of Psychology, vol. 30, no. 1-2, pp. 59-73, 2009.

[25] L. Fu, W. Song, W. Lv, and S. Lo, "Simulation of emotional contagion using modified SIR model: a cellular automaton 
approach," Physica A: Statistical Mechanics and Its Applications, vol. 405, pp. 380-391, 2014.

[26] D. Nilsson and A. Johansson, "Social influence during the initial phase of a fire evacuation-analysis of evacuation experiments in a cinema theatre," Fire Safety Journal, vol. 44, no. 1, pp. 71-79, 2009.

[27] Y. Mao, Z. Fan, J. Zhao, Q. Zhang, and W. He, “An emotional contagion based simulation for emergency evacuation peer behavior decision," Simulation Modelling Practice and Theory, vol. 96, Article ID 101936, 2019.

[28] W. O. Kermack and A. G. Mckendrick, "A contribution to the mathematical theory of epidemics," Proceedings of the Royal Society of London, Series A, Containing Papers of a Mathematical and Physical Character, vol. 115, no. 772, pp. 700-721, 1927.

[29] P. S. Dodds and D. J. Watts, "A generalized model of social and biological contagion," Journal of Theoretical Biology, vol. 232, no. 4, pp. 587-604, 2005.

[30] M. Cao, G. Zhang, M. Wang, D. Lu, and H. Liu, "A method of emotion contagion for crowd evacuation," Physica A: Statistical Mechanics and Its Applications, vol. 483, pp. 250-258, 2017.

[31] Q. Xiao and J. Li, "Evacuation model of emotional contagion crowd based on cellular automata," Discrete Dynamics in Nature and Society, vol. 2021, Article ID 5549188, 18 pages, 2021. 